\title{
CHRONIC MAXILLARY SINUSITIS IN CHILDREN
}

\author{
BY \\ J. F. BIRRELL \\ From the Ear, Nose and Throat Department, the Royal Hospital for Sick Children, Edinburgh
}

(RECEIVED FOR PUBLICATION APRIL 4, 1951)

There has been a considerable volume of literature on the subject of sinusitis in childhood during the past 30 years; much of it deals with the results obtained in routine proof puncture or antral lavage. For example, Mollison and Kendall (1922) found evidence of sinus infection in $22 \%$ of 102 routine tonsil and adenoid cases. Crooks and Signy (1936) reported 24 positive proof punctures in 100 routine tonsil and adenoid cases, while Gerrie (1939) puts the percentage at 29 in $\mathbf{3 0 0}$ such patients. Carrying this further, we find that Carmack (1931) reports positive findings in $14.2 \%$ of a series of 211 tonsil and adenoid cases from which he had excluded all known sinusitic and allergic patients. There are two reports of proof puncture results in known or suspected sinus cases. Crooks (1947) found evidence of infection in $59.6 \%$ of 570 cases with opaque antra seen during 1946, while Walker (1947) obtained positive findings in $76 \%$ of 442 cases with sufficient signs and symptoms to warrant puncture out of 1,779 tonsil and adenoid patients. Lastly, Ebbs (1938) has obtained post-mortem evidence of sinus infection in $30.6 \%$ of 496 routine necropsies.

It is small wonder that sinusitis has attracted considerable attention during this period, and the importance given to this condition is reflected in the space devoted to it in StClair Thomson's book. In the second edition, published in 1916, the condition is dismissed as rare, but in the current edition, published in 1948, several pages are given to it. The implications are obvious. Ebbs' figures suggest that nearly one-third of all children suffer from sinusitis, while other reports imply that one in every four or five children requiring removal of tonsils and adenoids also needs attention to the maxillary antra. If we accept this premise wholeheartedly, we must also be willing to accept the consequences, or be prepared to explain any anomalies in the deductions arising from this premise.

The first question to be answered is how chronic sinusitis arises. It is generally conceded that, in adults at least, chronic sinusitis does not arise per se, but develops from an acute maxillary sinusitis in which the infected material cannot, or does not, drain away. Among adults, many more people suffer from acute maxillary sinusitis than ever develop chronic sinusitis, as most cases of acute infection return to normal. It is natural to assume that in children there will be many more cases of acute maxillary sinusitis for the same reason. Yet it is generally stated that acute maxillary sinusitis in children is an uncommon condition, and the records of the E.N.T. Department of the Royal Hospital for Sick Children in Edinburgh tend to support this opinion, as during the past five years, when over 10,000 new patients were examined, only one case of acute maxillary sinusitis was seen. How is this to be explained ? It may be that, as with acute tonsillitis, the young child makes no complaint of infra-orbital pain, accepting it as part of an acute coryza, or ascribing it to toothache. If this be so, it is possible that the great majority of children suffer from acute maxillary sinusitis, which results in one child in every three, four or five having chronic maxillary sinusitis. Or again it may be that chronic maxillary sinusitis in children differs from the adult infection by arising per se in the antrum. Might it not be that chronic sinusitis arises from acute sinusitis, and is not as common as one is led to believe?

The second question to be considered is concerned with the fact that during childhood the maxillary sinus, in common with other sinuses, is in a state of active development. It grows to fill the maxilla as the teeth descend to the alveolar margin and finally erupt. If there is chronic infection lying close to these developing teeth, why is there no evidence of the eruption of unhealthy teeth, such as one finds after osteomyelitis of the maxilla ? And, again, taking as a corollary the development. of the mastoid air cells, why is there no arrested aeration and growth of the maxillary air sinus ? The lack of development of a normally cellular 
mastoid has been associated with the presence of otitis media in infancy and early childhood. Is one not justified in assuming that similar changes should be found in the antra of adults, and that one in every three, four, or five adults should show arrested development of the paranasal air cells, either unilaterally or bilaterally ?

Thirdly, if such a great percentage of children has been shown in the past $\mathbf{2 0}$ or $\mathbf{3 0}$ years to have had such a degree of sinusitis as to require energetic surgical correction, one must ask what happened to sinus infections in children in the past ? The treatment of otitis media in childhood is being prosecuted with zeal in the hope of materially reducing the numbers of adults with chronic otitis media. Will these energetic measures now adopted in the treatment of sinusitis in children similarly reduce the numbers of chronic sinusitis patients in 20 years? If so, the measures will be justified, but if not, one must assume that chronic sinusitis in childhood does not persist into adult life. In other words, so-called chronic sinusitis in children must be considered a self-limiting disease, thus differing from chronic infection elsewhere. If it is selflimiting, when does it clear up, and why? The opinion of Dean (1925) and others that removal of the adenoids and tonsils will cure $80 \%$ of cases of chronic sinusitis lends support to the theory that the natural regression of lymphoid tissue in the nasopharynx about puberty may determine this limitation of infection in the sinuses. The view is currently held in many quarters, on the other hand, that it is the sinusitis which causes and maintains the infection in, and the enlargement of, adenoids. If this view is correct, how can one explain either the disappearance of infection or the atrophy of the adenoids? I doubt if it can be explained on this hypothesis, unless infection does not, in fact, disappear, but is really very uncommon.

The impression has been gained in the operating theatre that true chronic maxillary sinusitis is indeed uncommon, and the case notes of all the proof punctures, 240 in all, performed between January 1, 1946, and October 31, 1949, were reviewed early in 1950. Arising from these findings certain investigations have since been made, and this report is concerned, firstly, with the results obtained, secondly with the investigations, and thirdly with a discussion of the problem in relation to the literature on the subject. It should, however, be emphasized that this paper does not represent a controlled or planned investigation, but is a bald report of the findings.

One other point should be stressed at this juncture. About half-way through the period under review, it occurred to me that as these cases awaited their turn on the ordinary tonsil list, the sinus infection might have undergone resolution between the date of diagnosis and the date on which the child was sent for. Accordingly, these patients were taken out of their turn at an extra operating session so that before the end of the series cases diagnosed as sinusitis were operated upon within a week or two. The result of this procedure did not, in any material way, alter the percentage of positive results, and the series may thus be treated as a whole.

\section{Amalysis of Case Notes}

Sex. Of 240 case sheets examined, 160 related to boys and 80 to girls, a ratio of boys to girls of 2:1.

Age. Only children under 12 years of age are admitted to this hospital, and the ages are shown in the accompanying graph. It will be seen that

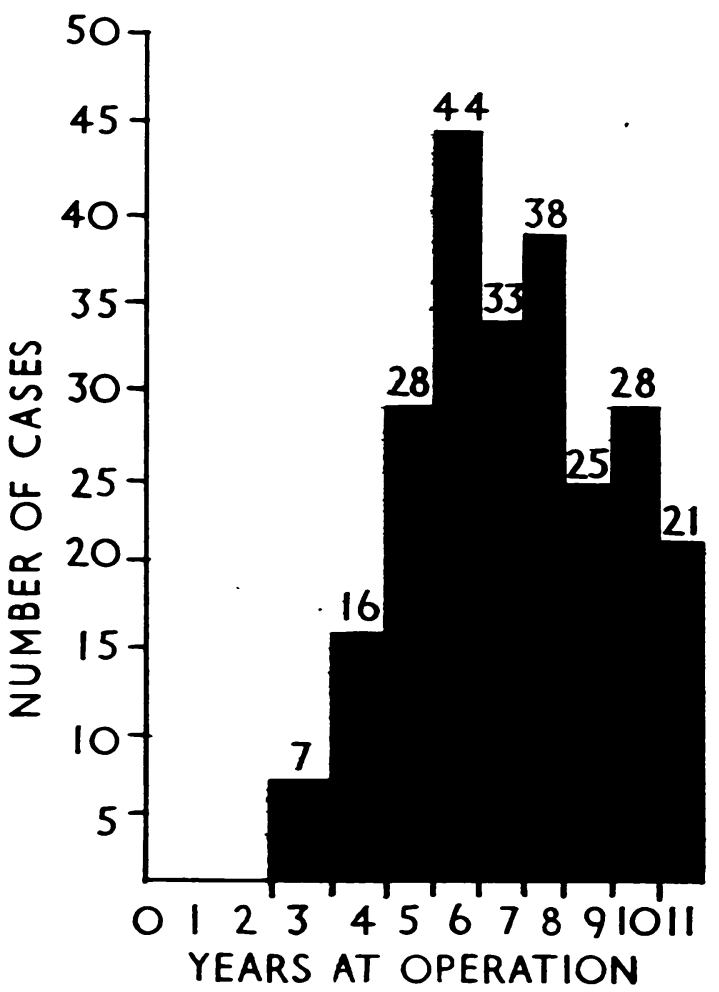

Fig. 1.-Graph showing age at puncture.

there is a preponderance of children in the 6-8 years age group. This is the age at operation, and the preponderance of this age group would be even more marked if cases were examined at the age at which sinusitis was diagnosed, as many of the 
9 to 12-year-old children had waited up to two years for admission.

Symptoms. It is very difficult in a review such as this to obtain an accurate picture of the presenting symptoms, as case histories have been taken by a number of different people-by residents, outpatient sisters, secretaries and surgeons. There is thus no unanimity of view, as would be obtained in a planned investigation, such as that of Birdsall (1939).

For what they are worth, the following symptoms have been noted: nasal catarrh, nasal discharge, or running nose (60 cases); frequent colds (40 cases); nasal obstruction or mouth breathing (35 cases); persistent cough ( 23 cases); bronchitis (10 cases); bronchiectasis (11 cases); headaches (19 cases); asthma (22 cases); hay fever (one case); allergic rhinitis (one case); discharging ears or deafness (27 cases); laryngitis (five cases); pharyngitis (one case); epistaxis (two cases); night sweats (one case); and acidosis (one case). In 39 case sheets there was insufficient history for the predominating symptom to be listed.

Signs. On examination of the nose, the findings have varied with the descriptive power of the examiner, and I have aggregated these into groups as follows: nose clear, i.e. a dry nose with no swelling of the turbinates ( 58 cases); congested nose (17 cases); allergic type of nose, i.e. pale, swollen turbinates (35 cases); nose containing unspecified discharge ( 50 cases); mucoid discharge (four cases); mucopurulent discharge (19 cases); purulent discharge (29 cases); crusted discharge (21 cases); and deflected septum (15 cases). No notes were made on eight case sheets.

Tonsils and Adenoids. Tonsils and adenoids were present in 117 cases, and had been removed in 117 cases. In the remaining six case sheets there are no notes. When tonsils and adenoids were present, their removal was carried out at the same time as the puncture in 42 cases. Of the patients whose tonsils had previously been removed, tonsillectomy was deemed inadequate in nine cases. Thirty-four patients were recommended to have adenoids (either the primary growth or the recurrence) removed at the same time as the puncture, and this is distinct from the $\mathbf{4 2}$ tonsil and adenoid operations listed above.

Radiology. Positive radiological findings were obtained in every case subjected to proof puncture, and no case was clinically labelled sinusitis until a radiograph revealed either mucosal thickening or opacity of the antrum. This aspect of the diagnosis will be discussed in greater detail later.

Technique. The children were all dealt with as in-patients. After pre-operative luminal the child was anaesthetized with ethyl chloride vapourized in a Guy's inhaler. No local cocaine anaesthesia was used. An opportunity was thus afforded of palpating the nasopharynx and removing any adenoid mass requiring surgery, and palpation and curettage is now part of the routine procedure of a proof puncture.

A Lichwitz trocar and cannula were used, and sterile saline solution injected into the antrum from a $5 \mathrm{ml}$. syringe carrying a blunt-pointed needle inserted through the cannula. A $2-3 \mathrm{ml}$. quantity of the solution was injected into the antrum, the head being rolled through $45^{\circ}$ to the corresponding side. The solution was then reaspirated into the syringe and examined macroscopically for mucopus or pus. If this were present, further wash-outs of this kind were used, or a Higginson's syringe was attached to the cannula, and the classical wash-out employed. With thls injection and reaspiration technique no accidents have occurred. This method is thought to be the only accurate means of determining the contents of an antrum, as the simple antral wash-out, without preliminary injection and aspiration, carries with it all secretions lying in the nasal cavities and nasopharynx, and, indeed, in bronchiectatic subjects, much pulmonary secretion is added to the specimen during the cough asscciated with recovery from anaesthetic. No reliance can be placed on the antral wash-out as a diagnostic guide. It is a therapeutic measure alone.

\section{Results}

One hundred and forty-two double punctures were carried out. The right antrum alone was punctured 51 times, and the left on 47 occasions. Thus, the 240 cases represent 382 punctures. The results are shown in Table 1.

Resolving the double proof punctures to single ones, the total number of antra in which pus was

TABLE 1

240 Cases Representing 382 Punctures

\begin{tabular}{|c|c|c|c|c|}
\hline & $\stackrel{\infty}{=}$ & 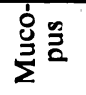 & 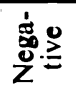 & ฐ \\
\hline $\begin{array}{l}\text { Double proof puncture ; same } \\
\text { reading in each }\end{array}$ & 8 & 2 & 109 & 119 \\
\hline $\begin{array}{c}\text { Double proof puncture ; left } \\
\text { negative ; right }\end{array}$ & 7 & 7 & & 14 \\
\hline $\begin{array}{c}\text { Double proof puncture : } \\
\text { negative, left } \ldots \\
\text {. . }\end{array}$ & 5 & 4 & & 9 \\
\hline Right proof puncture .. & 5 & 1 & 45 & 51 \\
\hline Left proof puncture & 3 & 1 & 43 & 47 \\
\hline Total & 28 & 15 & 197 & 240 \\
\hline
\end{tabular}


found is 36 , and the total number showing mucopus is 17, while a total of 329 antra gave a negative result. That is to say, $9 \cdot 42 \%$ of the antra contained pus, and $4.45 \%$ yielded mucopus. These results were found in a series of cases diagnosed clinically and radiologically as chronic sinusitis, and are not a series of routine punctures.

Analysing these results with respect to the symptoms and signs, two things must be borne in mind. In the first place many children had more than one symptom or sign, and this means that the result is marked opposite each. For example, a child with bronchiectasis, giving a history of frequent colds, who had pus in one antrum, would have the result shown against each of these symptoms. The second point to be noted is that the numbers in these tables refer to cases, not punctures, so that a bilateral puncture is shown as one case. The results are correlated with symptoms in Table 2, with signs in Table 3 and with the presence or absence of tonsils and adenoids in Table 4.

TABLE 2

Results Correlated WITH SyMptoms

\begin{tabular}{|c|c|c|c|c|c|c|c|}
\hline & & & & $\stackrel{\infty}{2}$ & 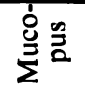 & 总き & छే \\
\hline Nasal catarrh & $\cdots$ & $\cdots$ & $\cdots$ & 10 & 3 & 47 & 60 \\
\hline \multicolumn{2}{|c|}{ Frequent colds .. } & $\cdots$ & . & 4 & 2 & 34 & 40 \\
\hline \multicolumn{2}{|c|}{ Nasal obstruction } & $\cdots$ & $\cdots$ & 1 & 2 & 32 & 35 \\
\hline \multicolumn{2}{|c|}{ Persistent cough } & $\cdots$ & . & 0 & 2 & 21 & 23 \\
\hline Bronchitis & $\cdots$ & $\cdots$ & . & 1 & 0 & 9 & 10 \\
\hline Bronchiectasis & $\ldots$ & $\cdots$ & $\cdots$ & 4 & 1 & 6 & 11 \\
\hline Headaches & $\cdots$ & $\cdots$ & $\cdots$ & 2 & 3 & 14 & 19 \\
\hline Asthma .. & $\cdots$ & $\cdots$ & $\cdots$ & 3 & 1 & 18 & 22 \\
\hline Hay fever & $\cdots$ & . & $\cdots$ & 0 & 0 & 1 & 1 \\
\hline Allergic rhinitis & & $\cdots$ & $\cdots$ & 1 & 0 & 0 & 1 \\
\hline Otorrhoea or & deaf & & $\cdots$ & 2 & 3 & 22 & 27 \\
\hline Laryngitis & $\cdots$ & $\cdots$ & $\cdots$ & 0 & 1 & 4 & 5 \\
\hline Pharyngitis & $\cdots$ & $\cdots$ & $\cdots$ & 0 & 0 & 1 & 1 \\
\hline Epistaxis & $\cdots$ & $\cdots$ & - & 1 & 0 & 1 & 2 \\
\hline Night sweats & $\cdots$ & . & . & 0 & 0 & 1 & 1 \\
\hline Acidosis .. & $\cdots$ & $\cdots$ & . & 0 & 0 & 1 & 1 \\
\hline No notes & $\cdots$ & $\cdots$ & $\cdots$ & 3 & 2 & 34 & 39 \\
\hline
\end{tabular}

TABLE 3

Results Correlated with Signs

\begin{tabular}{|c|c|c|c|c|c|c|c|}
\hline & & & & $\stackrel{\infty}{Z}$ & 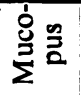 & 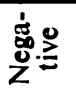 & 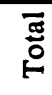 \\
\hline Clear & . & . & . & 3 & 4 & 51 & 58 \\
\hline Congestion & . & . & . & 4 & 0 & 13 & 17 \\
\hline Allergy .. & . & . & . & 1 & 0 & 34 & 35 \\
\hline Discharge, & peci & & . & 6 & 3 & 41 & 50 \\
\hline Mucoid dis & urge & $\cdots$ & . & 0 & 1 & 3 & 4 \\
\hline Mucopurule & disch & rge & $\cdots$ & 2 & 3 & 14 & 19 \\
\hline Purulent di & arge & . & $\cdots$ & 10 & 1 & 18 & 29 \\
\hline Crusted disc & rge & $\cdots$ & $\cdots$ & 3 & 1 & 17 & 21 \\
\hline Septal defle & & $\cdots$ & . & 1 & 1 & 13 & 15 \\
\hline No notes & $\cdots$ & $\cdots$ & $\cdots$ & 0 & 3 & 5 & 8 \\
\hline
\end{tabular}

A correlation of the results with symptoms and clinical features offers no striking solution, and no statistical analysis has been attempted in view of the lack of standardization in note-taking and examination. The first result calling for comment is that less than one-fifth of the cases suffering from ear trouble gave positive proof puncture findings. This surprised me, as I felt that chronic sinus disease played a larger part in maintaining ear trouble in children.

The other significant fact is that five of the 11 bronchiectatic patients had a positive puncture.

TABLE 4

Results Correlated with Presence or Absence of TONSILS AND ADENOIDS

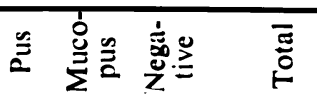

Tonsils and adenoids

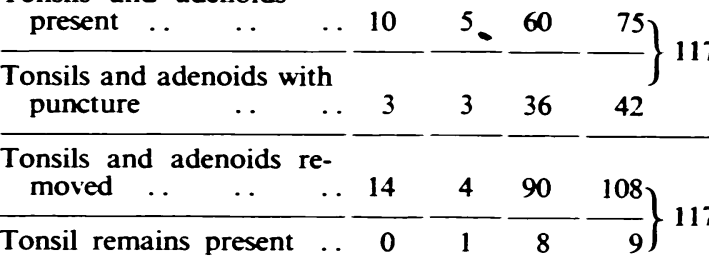

\section{Adenoids removed with}

$\begin{array}{ccccc}\text { puncture } & \ldots & \ldots & 1 \\ \text { No notes } & \ldots & \ldots & \ldots & 0\end{array} \frac{1}{1} \frac{32}{5} \frac{34}{6}$


This might be allied to the report of positive findings in 11 of the 29 patients showing pus in the nose on clinical examination. The association between chronic sinus infection and bronchiectasis is well recognized (Hogg, 1950). In all four cases of bronchiectasis with pus in the antrum, the nose showed purulent secretion, but, on the other hand, pus was present in the nose of two other patients suffering from bronchiectasis, in each of whom a bilateral puncture gave negative results.

There appear to be no other coincidences in the series, and one does not, therefore, seem justified in making any forecast of the probable result of proof puncture from an assessment of the symptoms and clinical signs. Nor, indeed, has experience shown that any reliable evidence can be obtained from radiographs. Positive and negative results have been found in cases whose radiographs show complete antral opacity or gross mucosal thickening. The appearances are not as diagnostic as in adults, for in children the results are confused by the growth of the antra, which are often shallow, and by the lack of air entry, either due to enlarged turbinates, enlarged adenoids, secretion lying in the nose, or habitual mouth breathing. The confusion in assessing radiographs was apparent in the cases of two children of the same age, both showing pus in the nose and both suffering from bronchiectasis, who had bilateral punctures performed on the same morning. The radiograph of one showed bilateral antral opacity, the other showed a moderate degree of mucosal thickening. The child with complete antral opacity had a bilateral negative puncture, while the other, with mucosal thickening, showed pus in each proof puncture !

\section{Follow-Up}

The punctures were repeated in the positive cases at an interval varying from one week to one month from the time of the initial puncture. The results of this puncture showed that four cases still produced pus in an antrum. Of these, two cleared up at further puncture, but two remained positive after repeated punctures and these were drained intranasally. Thus only two of the $\mathbf{3 8 2}$ in the series did not clear with proof puncture, and for this reason only these two might be said to have suffered from irreversible chronic maxillary sinusitis, a percentage of $0.53 \%$.

\section{Discussion}

These results do not bear comparison with those quoted in the literature. To find mucopus or pus in the antra of only $13.9 \%$ of a series of patients diagnosed clinically and radiologically as cases of chronic sinusitis compares strangely with the $59.6 \%$ of Crooks (1947) and the 76\% of Walker (1947). The obvious explanation is that the latter figures are those of antral wash-outs, and not of proof punctures.

Walker describes his operation as antral lavage, and Crooks, who quoted his figures during a discussion on a paper by Archer (1947), uses the expression ' washed out', though his earlier paper (Crooks and Signy, 1936) carefully described how the antral contents were aspirated. It has been explained that an antral wash-out is not a diagnostic test, and Walker's statement that the nose was blown on the morning of operation can hardly be taken as proof that abnormal secretions did not remain in the nasal cavities or nasopharynx. The average child is singularly unable or unwilling to clear the nose by blowing.

The problem of finding diagnostic criteria on which to pronounce a given sinus to be infected with any degree of certainty is, to my mind, an extremely difficult one. Dean (1925) thought diagnosis was easy, and other writers, such as Birdsall (1939) and Walker (1947), appear to rely on symptoms, signs and $x$-ray findings in their assumption that a child has sinusitis.

In most articles mention has been made of radiological findings, and $I$ have stated above that all the cases dealt with in this series had preliminary radiological evidence of antral opacity or of mucosal thickening, though those appearances bore no relation to the actual results. The earlier the paper, the greater appears to have been the faith in radiography. Cleminson (1921) considered radiographs valuable, while Dean (1925) stated that 'the greatest benefit to be derived from roentgen-ray examination is to tell us whether or not a given sinus is present, and, if present, whether on an anatomical basis, it has clinical significance '. Crooks and Signy (1936) judged radiography to be ' the most sensitive of all the diagnostic tests' because it 'will show slight and transitory changes of mucous membrane'. They go on to say that radiography may be misleading if it is not correctly interpreted and correlated with the clinical condition. They found, however, that it was common to wash out an antrum which was opaque to $x$-rays, and yet obtain a clear return, and concluded that the antrum is diseased none the less, but is draining adequately. I confess that I find it difficult to follow this argument in the light of my own experience, and $I$ tend to agree with Macbeth (1950) who believes that radiographs are of no use in determining the nature of mucosal changes in children.

Bowen-Davies (1938) made a bacteriological study of the results obtained in puncturing the antra of 55 children, and his results are rather astonishing. 
Of the 110 antra, 66 appeared, from x-ray examination, to be infected. Of these, 27 were proved to be bacteriologically unhealthy, and 39 were sterile. His conclusion is: 'It appears, therefore, that when the skiagram is positive the antrum will probably be sterile.' Of the $\mathbf{4 4}$ antra with negative radiographs, 35 were sterile and nine were infected, and his conclusions are: 'It is therefore probable that an antrum which appears normal on $\mathrm{x}$-ray will be sterile, but there is a possibility (one in five) that it may prove to be infected.' Bowen-Davies assesses radiographs as of little use in diagnosing infection in children.

If one cannot rely on a correlation of clinical and radiological features to diagnose with accuracy chronic sinus infection in children, one's thoughts turn to other diagnostic criteria of infection. Carmack (1931) has studied the blood picture in a series of cases, and he finds a moderate increase in white blood corpuscles, a decrease in the red cells and a low haemoglobin level. The blood chemistry is normal. These findings do not suggest that any assistance is to be had from a routine examination of the blood.

One of the investigations which leads one to suppose chronic infection to be present in the body is a high erythrocyte sedimentation rate. More recently we have been doing routine E.S.R. readings on proof puncture cases, but after obtaining such confusing results as bilateral thick pus in a child with an E.S.R. of $6 \mathrm{~mm}$. in the first hour, and a bilateral negative puncture in a child whose E.S.R. was $22 \mathrm{~mm}$. in the first hour, as well as a host of other results showing no definite trend of correlation, the procedure has been abandoned as a diagnostic aid.

Then a series of cases was investigated in the hope that one might explain the clinical and radiological findings on the basis of nasal allergy. It was not thought practicable to remove portions of mucous membrane for biopsy under the same anaesthetic (ethyl chloride) as served for the puncture and attention to tonsils and adenoids. Nor did we consider ourselves justified in turning the procedure into a major operation by having the child intubated for this purpose. Accordingly, nasal swabs were taken and examined for eosinophils with conspicuous lack of success, and the conclusion reached was that nasal allergy was not the explanation of the negative punctures in radiologically positive cases.

It would appear, therefore, that there is no means at present of diagnosing with any degree of certainty the contents of an antrum in childhood. One cannot foretell the cases in which pus or mucopus will be obtained at proof puncture. It therefore follows that, in order to avoid missing the few cases with evidence of infection in the antrum, every patient showing antral abnormality on radiographs must be proof punctured, and, until a reliable diagnostic criterion is discovered, this wholesale puncturing of antra must be continued as the only means of establishing diagnosis.

Children are apprehensive of anaesthetics, especially ethyl chloride, and of manipulations without general anaesthesia, and we should therefore be able to treat the occasional antrum showing pus at the same time as we arrived at the diagnosis. It appears from the results quoted above and from those of other writers that the vast majority of antra clear up after a single antral wash-out, and for this reason any antrum showing pus on aspiration has the sinus flushed out. Again, many authorities believe that the removal of tonsils and/or adenoids is essential to the cure of sinus infection, so to complete the cure these should be dealt with at the same operation and under the same anaesthetic.

The only author opposed to antral wash-out is Scott Stevenson (1947) who thinks that it causes otitis media. He agrees with Dean (1925), Cleminson (1921), Mollison and Kendall (1922) and Carmack (1931), among others, in advocating the removal of the adenoids, asserting that he has never seen a case of maxillary sinusitis in childhood in which the adenoids were not present, even when they were supposed to have been removed. This agrees with the experience of most operators, except Walker (1947) who found the tonsils more blameworthy than the adenoids, and assessed their degree of involvement by their actual size.

There is unanimity that most sinusitis in children can be cured by antral wash-out, with or without the removal of tonsils and adenoids, but, if this is so, was the sinusitis treated really chronic or irreversible, as it is currently described, at the time? Or was there merely some infected material which happened to be in the sinus? Crooks and Signy (1936) have declared that in the cases where a negative puncture appears to confound the radiological appearance, the sinus is actually infected, but is draining well. In view of the number of negative punctures in this series, I wonder whether those cases with transient pus or mucopus are not really cases of nasal catarrh where the sinus is draining poorly, and whether indeed the two antra persistently positive were not the only cases of true sinusitis in the whole series ?

Conservative measures advocated for the treatment of sinusitis include change of climate to the seaside (Dean, 1925; Crooks and Signy, 1936), Proetz displacement (Birdsall, 1939; De Boissière, 1949) and x-radiation (De Boissière, 1949). Dietetic therapy is recommended by Dean (1925), and this should include a liberal supply of vitamin $A$. 
Dietetic errors, especially excessive carbohydrate intake, have been mentioned by Scott Stevenson (1947) as combining with improper ventilation of the home to produce catarrhal changes. Macbeth (1950) uses postaural nasal soaks of freshly mixed penicillin, albucid and tuamine sulphate. The antibiotics may be instilled into the antra through indwelling catheters (Archer, 1947; Harpman, 1947) or polythene tubing (Hogg, 1950). Bateman (1945) suggested a paste of penicillin, flavazole and soluthiazole in lanette wax or concentrated plasma solution to the consistency of honey for use in adults, and Harpman (1947) comments on its use in children.

Should conservatism fail, or minor surgery not result in a cure, recourse is had to antral surgery. The operation of current choice appears to be the Caldwell-Luc or radical antrostomy (Crooks, 1947; Harpman, 1947; Scott Stevenson, 1947; Macbeth, 1950, and others) though Crooks in 1936 recommended intranasal drainage. Admittedly only a small percentage of cases required this operation, and it was only resorted to when other methods failed, but it seems a serious operation to undertake in a child of 4 years of age, the case reported by Harpman. At the Caldwell-Luc operation, it is reported that the mucosa shows changes in no way inferior to the changes found in adults with chronic sinus suppuration (Crooks, 1947). Yet an investigation by Bowen-Davies (1938) showed that while the average time of elimination of lipiodol from the antra in sterile cases was 17.9 days, the average time of elimination in infected cases was only 14.9 days. He explains this by the fact that there is reduced capacity in these antra, due to mucosal encroachment, but the function of the cilia is not disturbed by infection. It seems rather a radical procedure to remove ciliated epithelium which is performing its function normally.

This is the general picture of the approach to the problem in the country today, but I must confess to a certain scepticism. It appears that many of the deductions made arise from the assumption that sinusitis is an extremely common condition in children (Guthrie, 1939), an assumption which does not appear to me to be warranted. I would hardly go the length of Hajek whom Crooks and Signy (1936) quotes as saying: 'The fantastic reports of some American authors ... who describe an enormous number of empyemas observed in the earliest years of childhood ... . are to be regarded with the greatest suspicion, as their methods of examination give no assurance of the reliability of their results.

The articles from which I have quoted in this paper were written by eminent authorities, and their methods are described in considerable detail.
Because their results differ widely from those which have been obtained in Edinburgh, it does not necessarily follow that they are wrong. Indeed, the results of my series bear no comparison with any other published figures, except those concerning the Carmack (1931) series from which he had excluded all known sinus and allergy cases.

One criticism which has been made in discussing this series with some of my colleagues is that no bacteriological examination was made of the aspirated fluid, and thus many cases have been labelled negative which were really positive. The obvious retort is that mucopus or pus is the diagnostic criterion of infection, and that one can grow bacteria from many surfaces in contact with air even when these surfaces are not infected. A glance at the literature will confirm the confusion of thought in this criticism. Crooks and Signy (1936) found the same organisms, admittedly in differing proportions, in positive and negative punctures, and Bowen-Davies's (1938) bacteriological conclusions from his series complicate the picture still further.

The literature leaves us with the impression that chronic maxillary sinusitis is a common condition which affects one in every three, four, or five children, but which cannot be diagnosed with certainty by clinical findings, blood examination, E.S.R. readings, radiography, or bacteriological results from proof puncture, and this condition must be treated by removing adenoids and tonsils, by antral wash-outs, indwelling catheters for antibiotics, or, if all else fails, by a Caldwell-Luc operation, even though the ciliated lining of the antra is functioning normally.

The more one reads about this confusing subject, the more one wonders whether there is really any such disease as chronic sinusitis, or whether the symptoms and signs might not be explained by some other mechanism. There is no doubt that the sinus mucosa in a child is capable of considerable swelling, and that this swelling may persist for a time. There is also no doubt that children are susceptible to repeated acute infections of the upper respiratory tract, resulting in mucosal swellings. To get adequate drainage and resolution, ventilation of the sinus is necessary, but is prevented by blockage of the airway through the nasal cavity. The blockage in turn is augmented by a swelling of the mucosa of the turbinates, so that a vicious circle is set up, the swollen mucosa blocking the nasal airway and the lack of ventilation interfering with shrinking of the nasal and sinus mucosa. Thus, as from enlarged adenoids, habitual mouth-breathing develops. In time the nasal mucosa may settle, but each succeeding acute infection prolongs the time, and once mouth-breathing is established, sinus ventilation 
remains impaired, and the sinus mucosa remains thickened.

The reason why removing tonsils and adenoids helps these cases is not so much that infection is eliminated, as that, after removal, there is an increased chance that nasal respiration will be resumed, especially during sleep. If the child's room is well ventilated during the night, nasal breathing should be restored, but if there is insufficient fresh air, catarrh and mouth-breathing may persist. This would serve to explain why the greatest percentage of so-called sinusitis cases are found in the 6 to 8 years group, for this period embraces the physiological enlargement of tonsils and possibly of adenoids. Such enlargement will cause a sense of stuffiness, quite apart from the superadded acute infections.

Secretion lying in the nose, which the child is not taught to blow out properly, further impedes airway. The discharge is either present at the anterior nares, from which it is wiped, not blown, or is continuously sniffed backwards, and the nose is never completely cleared.

If one accepts these facts, it serves to explain why so many so-called sinusitis cases are relieved by removing tonsils and adenoids, by Proetz displacements, by mechanical douching, or antral lavage. It also serves to explain why no reliance is to be placed on clinical features, radiographs and proof puncture results. No sinus can remain free from secretion when the nasal cavity, with which it communicates, contains a plentiful supply of secretion. A certain amount will undoubtedly escape back into the nose from the antra, but some may be left in place, and would explain the paradoxical statement that the sinus is infected, but is draining well. The sinus is not infected. It merely receives a certain amount of spillage from the nasal cavity, and the cilia get rid of this quickly against an obstructed ostium. How much more easily would the cilia deal with the secretion if the nose were property aerated and cleaned.

The correct way to aerate a nose is not to use vasoconstrictor drops or sprays, nor to douche it out via the antrum each week under local or general anaesthesia, but to train the child in nose-blowing and nose-breathing. No quantity of ephedrine and no multiplicity of wash-outs will ever clear the nose of a child who is afraid to use it for breathing. One constantly meets children who have a clear nasal airway, free from secretion, and whose adenoids and tonsils have been adequately removed, but who approach panic when told to close their lips and breathe through their noses. It is ten times worse in those whose adenoids are present, and whose noses, lined by swollen mucosa, are filled with secretion.
In a discussion on an upper respiratory clinic for children held at the Royal Society of Medicine in 1945, Gwynne-Evans (1945) showed radiographs demonstrating how the failure of the palato-lingual reflex to close the faucial isthmus resulted in mouthbreathing, while an active palato-lingual reflex preserves nasal respiration. But the radiographs also showed that the antra were hazy in the child who breathed through the mouth, and clear in the child who used the nose for breathing.

In the same discussion Nove (1945) mentioned that relaxation of lingual musculature resulted in a low level of the hyoid bone, and stated that this was associated with mal-related jaws, post-nasal catarrh and respiratory abnormalities. After orthodontic treatment the tone of the muscles improved, the hyoid assumed its normal level, and the tongue ceased sagging. This resulted, among other things, in an improvement in respiration. Again, the radiographs showed blurring of the antra before treatment, and clear antra thereafter.

These two papers lend unwitting weight to the theory that the clinical and radiological appearances of sinusitis in children may be found with mouthbreathing, and will disappear when nasal respiration is established. If, then, we disabuse our minds of the idea that chronic sinusitis in children is common, though undiagnosable, and search for one factor which will produce all the signs and symptoms associated with the condition, we find the answer in mouth-breathing. The treatment, therefore, should not be directed against a non-existent sinusitis, but against mouth-breathing. The children and their parents must be taught the importance of using the nose for its proper function. The children must be shown that it is possible to breathe adequately with the lips shut. Physiotherapists may have to issue the initial instructions on breathing exercises, and I venture to think that if these exercises are continued until nose-breathing has become unconscious, the clinical features will disappear. It may be necessary to remove adenoids and tonsils in the first instance, and to use drops of $2-5 \%$ argyrol to reduce any rhinitis, but these may be quickly dispensed with, and the dying habit of ' nose-drill ' continued.

In this way we may find that the so-called chronic sinusitis is really as uncommon as acute sinusitis in children, and that the figures of $0.53 \%$ of all so-called sinusitis cases is a correct one.

\section{Coniclusions}

The results of 382 proof punctures in $\mathbf{2 4 0}$ children, clinically and radiologically diagnosed as chronic sinusitis, showed positive results in $13.87 \%$, but 
only two children $(0.53 \%)$ were considered to be true cases of chronic maxillary sinusitis. An examination of the literature in conjunction with these results leads one to the conclusion that the condition cannot be diagnosed with accuracy by any means at our disposal. It is felt that the generally accepted theory that sinusitis is common in children is false, and does not withstand critical examination. If the problem is approached with an unbiased mind it will be appreciated that identical clinical and radiological findings may be produced by habitual mouth-breathing. Treatment should be directed in the first instance towards re-establishing nasal respiration rather than against a non-existent sinus infection.

A proportion of the cases reported in this paper were punctured by my senior colleague, Dr. C. E. Scott, to whom I am indebted for permission to include them in the series.

\section{REFERENCES}

Archer, G. E. (1947). Proc. R. Soc. Med., 40, 854.

Bateman, G. H. (1945). J. Laryng., 60, 110.
Birdsall, S. E. (1939). Ibid., 54, 549.

Bowen-Davies, A..(1938). Proc. R. Soc. Med., 31, 1411.

Carmack, J. W. (1931). Ann. Otol., etc., St. Louis, 40, 515.

Cleminson, F. J. (1921). J. Laryng., 36, 505.

Crooks, J. (1947). Proc. R. Soc. Med., 40, 857. and Signy, A. G. (1936). Archives of Disease in Childhood, 11, 281.

Dean, L. W. (1925). J. Amer. med. Ass., 85, 317.

De Boissière, V. (1949). Canad. med. Ass. J., 60, 14.

Ebbs, J. H. (1938). Brit. med. J., 1, 385.

Gerrie, J. (1939). Ibid., 2, 364.

Guthrie, D. (1939). Ibid., 2, 363.

Gwynne-Evans, E. (1945). Proc. R: Soc. Med., 38, 536.

Harpman, J. A. (1947). Brit. med. J., 2, 1054.

Hogg, J. C. (1950). Proc. R. Soc. Med., 43, 1089.

Macbeth, R. (1950). Irish J. med. Sci., no. 296, p. 362.

Mollison, W. M. and Kendall, N. E. (1922). Guy's Hosp. Rep., 72, 225.

Nove, A. A. (1945). $\quad$ Proc. R. Soc. Med., 38, 540.

Stevenson, R. Scott (1947). Ibid., 40, 858.

Walker, F. M. (1947). Brit. Med. J., 2, 908. 